\title{
Guest editorial: Special Issue on Science of Wear
}

\author{
Yonggang MENG ${ }^{1}$, Valentin L. POPOV ${ }^{2}$, Jean-François MOLINARI ${ }^{3}$ \\ ${ }^{1}$ State Key Laboratory of Tribology, Tsinghua University, Beijing 100084, China \\ ${ }^{2}$ Technische Universität Berlin, Institute of Mechanics, Berlin 10623, Germany \\ ${ }^{3}$ Civil Engineering Department, Materials Science Department, École Polytechnique Fédérale de Lausanne, Lausanne 1015, Switzerland \\ (C) The author(s) 2018. This article is published with open access at Springerlink.com
}

Among the basic tribological phenomena of contact, adhesion, friction, lubrication and wear, wear remains the least scientifically understood. At the same time, wear remains one of the most important tribological phenomena in practice, affecting all aspects of our lives and current technologies. The impact of wear is remarkable in terms of energy losses, environmental impact, component lifetime and economic costs. It may result in catastrophic failures and operational breakdowns that can adversely impact productivity and hence cost. Thus, controlling wear and process optimization play significant roles in sustainable development.

Our understanding of the fundamental processes of wear has been improving recently due to new theoretical approaches, improved experimental techniques and rapidly growing numerical capabilities. It is felt that now it is the right time to revisit the subject of wear intensively, with a special focus on the understanding of physical mechanisms. The Special Issue "Science of Wear" of Friction serves to survey the state-of-the-art in the field of wear research and to map out likely developments in the near future. Due to extreme complexity of the wear process, which includes processes from atomic scale up to the scale of the machine as a whole, such a survey cannot be complete. This issue represents exemplary topics which aim to provide a rough general picture of the temporary state of the science of wear and some of its challenges and tendencies.

The Special Issue consists of one review article, seven original research papers and a short communication. It covers a great variety of topics and methodological approaches in science of wear including adhesive wear, the influence of lubricants on wear, self-lubricating materials, the role of phase transformations and internal damping in the wear process, and the kinetics of the formation and wearing of tribological layers. Methodologically, the papers of the issue cover statistical correlation analysis, numerical simulations on atomic, mesoscopic and macroscopic scales, and experimental investigations on the mechanical system as a whole as well as on individual micro contacts.

The Issue is opened with a review article "Adhesive wear mechanisms uncovered by atomistic simulations". It is devoted to a review of a recent series of papers on adhesive wear which offers a new paradigm for future wear research. Starting with the breakthrough publication by Aghababaei, Warner and Molinari in Nature Communications (Nat Commun 7: 11816, 2016), this series developed a very promising approach for numerical simulations of adhesive wear. A central idea of the approach is the interplay of plasticity and adhesion which introduces a characteristic length scale governing the process of adhesive wear. The review also contains an extensive historical review of the main attempts in simulating wear.

The original research paper "Adhesive wear and particle emission: Numerical approach based on asperity-free formulation of Rabinowicz criterion" is closely related to the ideas described in the opening review paper as well as to an old idea of Ernest Rabinowicz about the criterion of looseness of wear particles. The paper discusses the key problem of those wear criteria which use the concept of "micro

Guest Editors: Yonggang MENG, E-mail: mengyg@tsinghua.edu.cn; Valentin L. POPOV, E-mail: v.popov@tu-berlin.de; Jean-François MOLINARI, E-mail: jean-francois.molinari@epfl.ch 
contact" or "asperity". For real fractal surfaces, this notion is very hard to define. The authors introduce a formulation which allows to completely avoid the notion of "asperity" and illustrate the application of this new approach by a series of wear simulations based on the boundary element method. One of the important conclusions of the paper is a possible breakdown of Archard's law of wear.

In the paper titled "Combined effect of boundary layer formation and surface smoothing on friction and wear rate of lubricated point contacts during normal running-in processes", the authors take a closer look at the running-in-process as essential stage of a lifetime of any tribological system. The authors carry out an experimental investigation and use stop-and-go experiments in combination with measurements of surface topography for discriminating the effects of boundary layer of change of surface topography.

In the research paper "Estimating anti wear properties of esters as potential lubricant-based oils using QSTR models with CoMFA and CoMSIA", the authors apply statistical regression analysis to correlate the structural properties of 57 esters and the wear properties of the oils based on these esters. This method allows considering much more governing parameters then is usually possible in any model based approach, thus producing a rough but complete picture of influence tendencies.

In the paper "Finite element simulation and experimental test of the wear behavior for selflubricating spherical plain bearings", the authors numerically and experimentally investigate wear in spherical plain bearings. They identify the stages of rapid initial and stationary wear. Through comparison of experimental findings with finite element simulation, the authors determine parameters of the wear law, which differs from the Archard's law and is formulated in terms of power dependencies of the wear rate on the normal pressure and sliding velocity.

In the paper "Effect of oxide film on nanoscale mechanical removal of pure iron", properties of an oxide film formed on a pure iron surface after being polished with an $\mathrm{H}_{2} \mathrm{O}_{2}$-based acidic slurry were investigated using an atomic force microscope (AFM),
Auger electron spectroscopy (AES), and angle-resolved X-ray photoelectron spectroscopy (AR-XPS) to partly reveal the material removal mechanism of pure iron during chemical mechanical polishing (CMP). The authors come to the conclusion that chemical corrosionenhanced mechanical wear may dominate the CMP process.

The paper "Single asperity friction in the wear regime" studies wear and friction and their relation by atomic scale simulations. The authors come to the conclusion that depending on adhesion parameters and load, regimes of atomic wear and plastic wear may occur. Interestingly, in the region of atomic wear, the well-known Amontons' law is valid even at the level of single asperities. It would be interesting to compare these findings with the old ideas of Holm and Rabinowicz about the existence of a very mild wear via atom-by-atom removal.

The research paper "Acoustic emission characterization of sliding wear under condition of direct and inverse transformations in low temperature degradation aged yttria-stabilized tetragonal zirconia polycrystalline (Y-TZP) and Y-TZP-Al2O3" is devoted to an experimental characterization of acoustic emission as a powerful in-situ tool for investigating the micromechanical process going on during the wear process. The paper combines an experimental investigation with corresponding simulations by the method of movable cellular automata.

The Special Issue is closed by a short historical review of the work of Ernest Rabinowicz on adhesive wear. Rabinowicz was one of the first tribologists who recognized the importance of adhesion in tribological contacts and formulated a number of key concepts in tribology. One of his fundamental ideas on the looseness of wear particles, Rabinowicz published in a short paper in 1958. On occasion of the $60^{\text {th }}$ anniversary of this work, the authors carried out a historical research of the subsequent development of the ideas of Rabinowicz and their impact on research in tribology and materials science. The communication is based both on a literature search and personal experience of one of the authors who was fortunate enough to be one of the $\mathrm{PhD}$ students of Ernest Rabinowicz at MIT. 